\title{
Optimization Studies of a Three-Stage Light Gas Gun
}

\author{
Lewis A. Glenn
}

This paper was prepared for submittal to the

American Physical Society

Topical Conference on Shock Compression of Condensed Matter

Amherst, $M A$

July 27-August 1, 1997

June 1997

This is a preprint of a paper intended for publication in a journal or proceedings. Since changes may be made before publication, this preprint is made available with the understanding that it will not be cited or reproduced without the permission of the author. 


\section{DISCLAIMER}

This document was prepared as an account of work sponsored by an agency of the United States Government. Neither the United States Government nor the University of California nor any of their employees, makes any warranty, express or implied, or assumes any legal liability or responsibility for the accuracy, completeness, or usefulness of any information, apparatus, product, or process

disclosed, or represents that its use would not infringe privately owned rights. Reference herein to any specific commercial product, process, or service by trade name, trademark, manufacturer, or otherwise, does not necessarily constitute or imply its endorsement, recommendation, or favoring by the United States Government or the University of California. The views and opinions of authors expressed herein do not necessarily state or reflect those of the United States Government or the University of California, and shall not be used for advertising or product endorsement purposes. 


\title{
OPTIMIZATION STUDIES OF A THREE-STAGE LIGHT GAS GUN
}

\author{
Lewis A. Glenn \\ Lawrence Livermore National Laboratory, Livermore, California 94550
}

\begin{abstract}
A new gasdynamic launcher is described, in which intact projectiles weighing at least 1 gram can be accelerated to mass velocities of $15-20 \mathrm{~km} / \mathrm{s}$. The system employs a conventional 2--stage light gas gun, with the barrel modified and filled with helium to act as a pump tube for a third stage. The key feature of the launcher is that the peak pressure in the third stage can be maintained below $2.5 \mathrm{GPa}$, thus assuring high efficiency and the integrity of the projectile.
\end{abstract}

\section{INTRODUCTION}

The maximum velocity attainable in a gasdynamic launcher is limited by the maximum sound speed in the driver gas. For a conventional 2 -stage light gas gun, the limit is $\sim 10 \mathrm{~km} / \mathrm{s}$. To achieve higher velocities requires adding additional stages. Staging methods have been reported whereby a pusher of high shock impedance impacts a stack of target plates in which the impedance decreases from plate to plate in the desired direction of motion $(1,2)$, and velocities up to $14 \mathrm{~km} / \mathrm{s}$ have been obtained in this manner. Chhabildas and his colleagues at Sandia National Laboratory have reversed this strategy with a graded density pusher, and they have demonstrated velocities up to $15.8 \mathrm{~km} / \mathrm{s}$ with a $6 \mathrm{~mm}$-diameter $\times 0.56 \mathrm{~mm}$ thick titanium projectile (3). A difficulty with this method is that the loading pulse is applied in a very short period of time and to achieve such a high velocity in this period requires a very high pressure, up to $100 \mathrm{GPa}$ or even more. This means that the projectile thickness must be kept very thin to prevent spall fracture, and the impactor design is highly constrained to avoid shock-melting or even vaporizing the projectile. Also, such high pressures effect large energy losses via $p d V$ work on the walls of the launcher; in the $15.8 \mathrm{~km} / \mathrm{s}$ experiment cited above, the kinetic energy efficiency of the third stage (ratio of the kinetic energy of the flyer to that of the impactor) was only $0.6 \%$.

It may be possible to avoid many of these problems by slightly modifying the configuration of the conventional 2-stage system, as shown in Fig. 1. Normally, a vacuum is drawn in the $2^{\text {nd }}$ stage launch tube of the gun, so that after the burst diaphragm is broken by the compressed hydrogen, the $2^{\text {nd }}$ stage projectile is accelerated with no downstream resistance. If instead, this launch tube is closed at the downstream end with a second burst diaphragm, and filled with an appropriate mass of light gas, the second-stage projectile will act to compress the gas in much the same manner as does the piston in the first stage. The third stage projectile is placed downstream of the second burst diaphragm in a smaller diameter barrel, which now becomes the final launch tube.

We are interested in the solution of the following problem: Given the fixed characteristics of a two-stage light gas gun (pusher mass and velocity, $m_{2}$ 


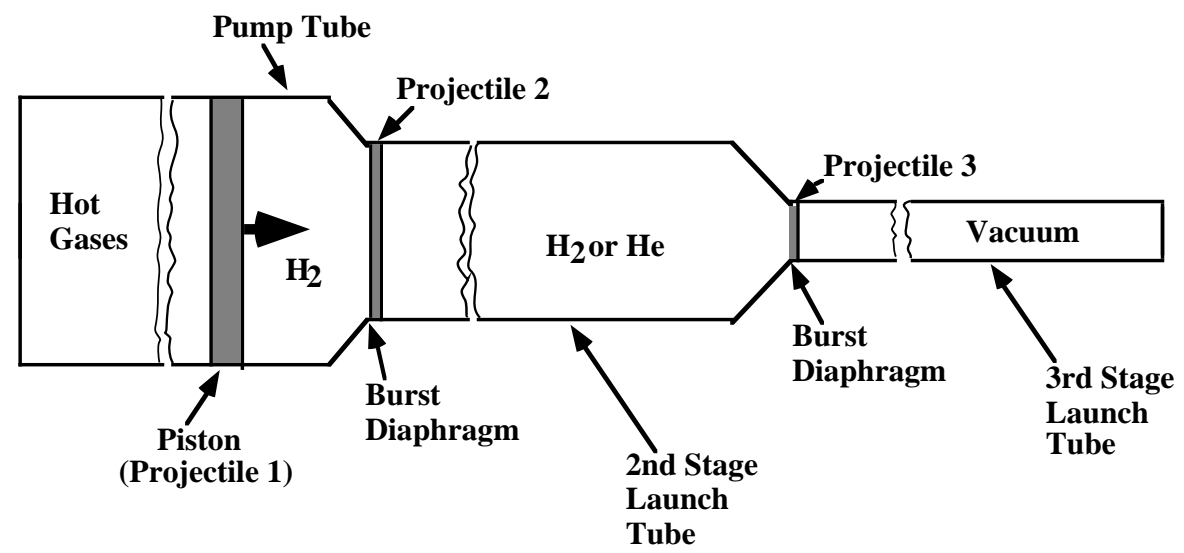

FIGURE 1. Sketch of the three-stage light gas gun.

and $v_{2}$, cross sectional area and length of barrel, $A_{2}$ and $L_{2}$ ), find the largest projectile mass, $m_{3}$, that can be accelerated to a final velocity, $v_{3}$, subject to the constraint that the maximum pressure on the projectile, $\hat{p}$ is less than some prescribed value. It is required to also find the corresponding third stage characteristics, $A_{3}$ and $L_{3}$, and the interstage gas mass, $m_{\mathrm{g}}$. Under certain simplifying assumptions, it can be shown that an exact solution to the above-posed problem exists (4). Our approach in what follows will be to use the analytical results to derive the approximate solution space and then to perform hydrodynamic code calculations to assess the effects of removing the simplifying assumptions.

\section{ANALYTICAL MODEL}

If it is assumed that 1) the walls of the gun tubes remain rigid, 2) there is a step change in cross section between stages and the pusher mass, $m_{2}$, is brought to rest precisely at this location, 3) pressure gradients in the interstage gas can be ignored, 4) a monatomic $\gamma$-law equation of state describes the gas, and 5) after the first shock, the gas is effectively isentropically compressed, then the following solution for the third stage mass is derived in (4):

$$
m_{3}=\frac{4 k}{3} \frac{m_{g}}{g^{2}}\left[\left(1+\frac{1}{\alpha}\right)-\frac{1}{k}\left(\frac{\Phi}{1+\Phi}\right)\right]
$$

where $g$ is the velocity gain, $v_{3} / v_{2}, k$ is the fraction of the peak internal energy of the gas that goes into increasing the kinetic energy of the projectile from its value at the time of peak pressure to its final value; $k$ is essentially a free parameter, however, choosing a value of $k$ much larger than 0.75 leads to diminishing returns, the energy saved being at the expense of greatly increased barrel length. The quantity, $\alpha$, derives from solution of the equation:

$$
\left(\frac{1+\alpha}{\alpha}\right)^{5 / 2} \frac{\alpha(1-\alpha)}{1-\alpha+[\alpha(1+\alpha)]^{1 / 2}}=\frac{3 A_{2} L_{2}}{4} \frac{\hat{p}}{m_{2} v_{2}^{2}}(2)
$$

All the quantities on the right-hand side of equation (2) are given, so that $\alpha$ is readily evaluated. The quantity, $\Phi$ in equation (1) is then found from the relation:

$$
\Phi=\frac{[\alpha(1+\alpha)]^{1 / 2}}{1-\alpha}
$$

and the gas mass, $m_{\mathrm{g}}$, is determined by:

$m_{g}=\frac{3}{4} m_{2}\left(\frac{\alpha}{1+\Phi}\right)$

The cross sectional area of the third stage launch tube, $A_{3}$, is then found from the relation:

$$
A_{3}=\left(A_{2}^{2} \Phi \frac{m_{3}}{m_{2}}\right)^{1 / 2}
$$


and finally, the length of the third stage launch tube, $L_{3}$, is given by:

$$
L_{3}=\frac{\left(\frac{1+\alpha}{1+\Phi}\right) \frac{m_{2} v_{2}^{2}}{\hat{p}}}{3 A_{3}(1-k)^{3 / 2}}
$$

To illustrate, the intermediate LLNL two-stage light gas gun has been shown to fire a projectile, $m_{2}=37 \mathrm{~g}$ at a velocity $v_{2}=6.2 \mathrm{~km} / \mathrm{s}$. The launch tube bore diameter is $D_{2}=28 \mathrm{~mm}$ (so that $A_{2}=6.16 \mathrm{~cm}^{2}$ ) and the length is $L_{2}=9 \mathrm{~m}$. Assuming it is desired that the projectile velocity from the added third stage be $v_{3}=20 \mathrm{~km} / \mathrm{s}$, $g=3.23$. If the third stage projectile is made of titanium alloy $\left(\rho_{3}=4.51 \mathrm{~g} / \mathrm{cm}^{3}\right)$, we choose $\hat{p}$ to be $2.5 \mathrm{GPa}$, less than half the $\sim 5.5 \mathrm{GPa}$ spall strength. Equations (1)-(6) then yield $\alpha=0.267$, $\Phi=0.793, m_{3}=1.65 \mathrm{~g}$, and $A_{3}=1.158 \mathrm{~cm}^{2}$, so that the bore diameter of the third stage barrel is 12.1 $\mathrm{mm}$ and the projectile length is $m_{3} / A_{3} \rho_{3}=3.16 \mathrm{~mm}$. Also, $m_{\mathrm{g}}=4.13 \mathrm{~g}$, which leads to a fill pressure of $0.46 \mathrm{MPa}$ assuming helium is employed, and $L_{3}=9.3 \mathrm{~m}$. Although this represents a quite acceptable parameter set, it might be desirable to reduce the barrel length. This could be accomplished by reducing the projectile mass (length) somewhat. Another alternative is to accept a slightly lower final projectile velocity. Code calculations show that the $1.65 \mathrm{~g}$ projectile attains $0.95 v_{3}$ in the first 3.9 $\mathrm{m}$, and is already at $0.9 v_{3}$ in $2.7 \mathrm{~m}$.

We have not accounted for losses thus far. These are from three principal sources, gasdynamic drag, heat loss to- and $p d V$ work done by the gas on the walls. We intend to choose $\hat{p}$ well below the spall strength of the projectile material, so as to minimimize $p d V$ work on the walls. Heat loss by radiation transport can be shown to be insignificant as long as the gas temperature remains below $\sim 10 \mathrm{eV}$. Gasdynamic drag and convective heat transport from the turbulent boundary layer adjacent to the wall are discussed below.

\section{HYDROCODE CALCULATIONS}

GGUN2 is an extension of earlier codes (GGUN(5) and $\operatorname{IGUN}(6,7))$ employed to study the performance of the two-stage light gas gun and various implosion and multistage launchers. It is an arbitrary Lagrange-Eulerian (ALE) code that solves the equations of motion in one dimension, but takes into account arbitrary flow cross section (including discontinuities), convective heat transport to the walls, and gasdynamic wall drag. Tabular equations-of-state can be employed, as well as Grüneisen, polynomial, and $\gamma$-law models, and JWLexplosive and Noble-Abel propellant burn packages have recently been added. There is also a coupled wall motion algorithm that allows the gun-barrel walls to move in response to the developing internal pressure. The equations of motion and other details have been described earlier (7).

The GGUN2 code was validated by comparing the computational results with experimental data from the first two stages of the three-stage light gas gun discussed in the previous section; it was shown in (4) that excellent agreement was obtained. Excellent agreement was also obtained by comparing third stage calculations with $2 D$ simulations performed with the CALE code (8).

Helium is the preferred working fluid for all stages beyond the second because a higher final sound speed can be produced for given initial and final pressures. This is true also for the second stage. For example, when we recalculated the two-stage launcher discussed above, using helium in place of hydrogen (all other parameters remained the same, including the initial gas density and temperature), the final velocity increased by about 3\%, notwithstanding the lower atomic mass of the hydrogen. However, practical considerations preclude the use of helium in the second stage. Although the velocity was slightly higher, the peak gas temperature in the AR (transition) section with helium was $6360 \mathrm{~K}$ compared with $1880 \mathrm{~K}$ with hydrogen. The slight increase in performance is more than countered by the potentially severe erosion that would be engendered by repeated use of the gun with helium. In the third stage, however, this is no longer a problem since the barrel in this stage 
cannot be salvaged and must be replaced after each shot.

Figure 2 shows the performance when the results of the analytical model are used to design the third stage. Both the velocity of, and the peak pressure on, the third stage projectile are shown for 3 cases. All 3 calculations employed tabular equations of
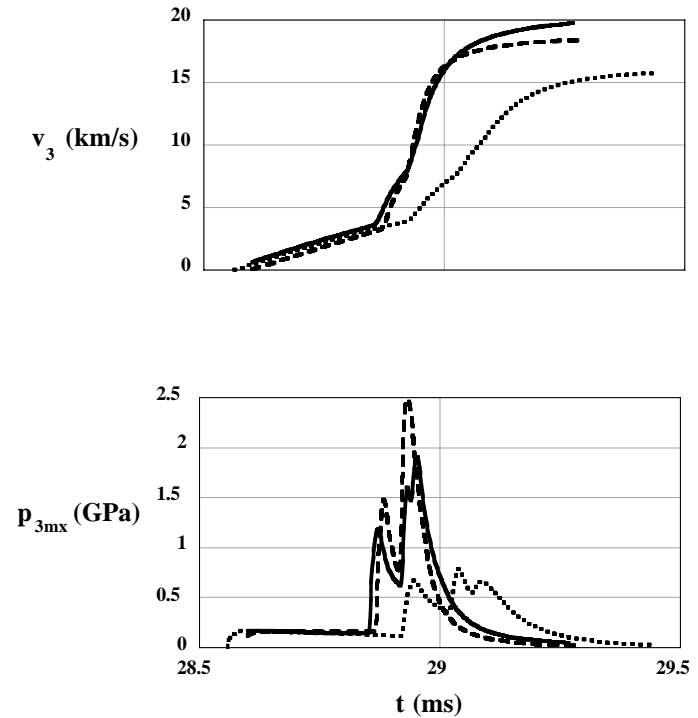

FIGURE 2 . Calculated velocity of and peak pressure on third stage projectile. Solid curve includes radial wall motion, dashed curve adds convective heat transfer, and dotted curve adds gasdynamic drag.

state for both the hydrogen in the second stage and the helium in the third. The solid curves depict the results when wall motion is included, but not heat transfer or drag. Assuming fully developed turbulent flow, the dashed curves include convective heat transfer from the helium plasma to the walls, and the dotted curves include both heat transfer and gasdynamic drag.

\section{DISCUSSIONS \& CONCLUSIONS}

The calculated results exhibit the effects of all important loss mechanisms, $p d V$ work on the walls (kept small by maintaining relatively low pressures in the system), heat transfer, and turbulent drag. The latter effect appears most important, and reduces the maximum velocity from 20 to $16 \mathrm{~km} / \mathrm{s}$. At the same time, the peak pressure on the projectile drops from $2.5 \mathrm{GPa}$ to approximately $0.8 \mathrm{GPa}$. Subsequent calculations have shown that shaving the length of the projectile to decrease the mass to $1 \mathrm{~g}$ (all other parameters fixed, and all loss mechanisms activated) increases the predicted velocity to 18 $\mathrm{km} / \mathrm{s}$ and decreases the peak pressure to less than 0.5 Gpa.

\section{REFERENCES}

1. Laptev, V. I., and Trishin, Yu. A., "Increase of initial velocity and pressure upon impact on an inhomogeneous target", Zh. Prikladnoi Mekhaniki i Tekhnickeskoi Fiziki, 6, 128-132 (1974); Eng. transl. Sov. J. Appl. Mech. and Tech. Phys., p. 837-841 (1976).

2. Fowles, G. R., Leung, C., Rabie, R., and Shaner, J., "Acceleration of flat plates by multiple staging", High--Pressure Science and Technology, Sixth AIRAPT Conference -1977, 911919, Plenum Press, New York (1979).

3. Chhabildas, L. C., Kmetyk, L. N., Reinhart, W. D., and Hall, C. A., Int. J. Impact Engrg., 17, 183194 (1995).

4. Glenn, L. A., "On how to make the fastest gun in the West", International Workshop on New Models and Numerical Codes for Shock Wave Processes in Condensed Media - 1997, Oxford, UK (see also Lawrence Livermore National Laboratory Report UCRL-JC-126057, January 1977).

5. Glenn, L. A. "Performance analysis of the twostage light gas gun", Shock Waves in Condensed Matter - 1987, 653-656, Elsevier Science Publishers, The Netherlands (1988). (see also Lawrence Livermore National Laboratory Report UCRL-96021, May 1987).

6. Glenn, L. A., Latter, A. L., and Martinelli, E. A., "Multistage gasdynamic launchers", Shock Compression of Condensed Matter - 1989, 977984, Elsevier Science Publishers, The Netherlands (1990). 
7. Glenn, L. A., Int. J. Impact Engrg., 10, 185-196 (1990).

8. Tipton, R., "CALE - a $C$-Language, arbitrary Lagrange Eulerian hydrocode for UNIX systems", Lawrence Livermore National Laboratory Report, to be published.

This work was performed under the auspices of the U.S. Dept. of Energy at LLNL under contract no. W-7405-Eng-48. 


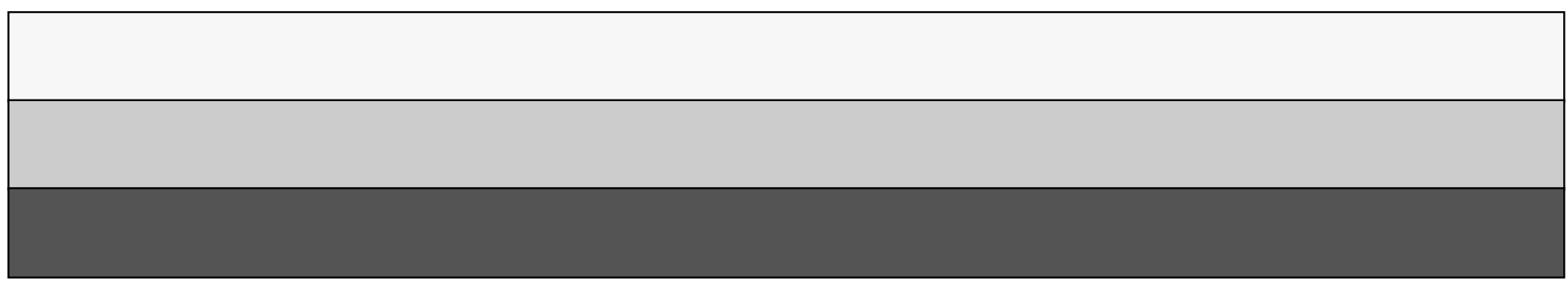

